\title{
Laparoscopic vs. open abdominal surgery in male pigs: Marked differences in cortisol and catecholamine response depending on the size of surgical incision
}

\author{
Angeliki Krikri, ${ }^{1 *}$ Vasileios Alexopoulos, ${ }^{1 *}$ Emmanouil Zoumakis, ${ }^{1,2}$ \\ Paraskevas Katsaronis, ${ }^{3}$ Evangelos Balafas, ${ }^{1}$ Gregory Kouraklis, ${ }^{3}$ \\ Panagiotis E. Karayannacos, ${ }^{1}$ George P. Chrousos, ${ }^{1,2}$ Gregory Skalkeas ${ }^{1}$
}

${ }^{1}$ Center of Experimental Surgery, Biomedical Research Foundation, Academy of Athens, ${ }^{2}$ First Department of Pediatrics, Athens University Medical School, Aghia Sophia Children's Hospital, ${ }^{3}$ Second Department of Propedeutic Surgery, Athens University Medical School, Laiko General Hospital, Athens, Greece

*Both authors contributed equally to this work

\begin{abstract}
OBJECTIVE: Minimally invasive operations, such as laparoscopic cholecystectomy and adrenalectomy, result in a more rapid recovery of normal function, less physiological disturbances and less stress to the organism than similar open operations. The purpose of this study was to determine the stress response associated with minimally invasive abdominal surgery compared to conventional small or large incision laparotomy. METHODS: We compared the responses of the stress hormones cortisol and the catecholamines adrenaline and noradrenaline to elective conventional and laparoscopic cholecystectomy and unilateral adrenalectomy in male pigs. Blood samples were taken from all animals at the same time, one day before surgery, at the beginning of the operation, every 15 minutes during surgery and on the first postoperative morning. RESULTS: Plasma adrenaline and noradrenaline concentrations were significantly lower in both cholecystectomies $(\mathbf{p}<\mathbf{0 . 0 5})$ and adrenalectomies $(\mathbf{p}<0.01)$ during laparoscopic than during open surgery. Plasma cortisol levels were significantly lower in laparoscopic than in open adrenalectomies both during surgery and on postoperative day one $(p<0.05)$, while no major differences in cortisol levels were observed between laparoscopic and open cholecystectomies. Thus, the stress-related benefit of laparoscopic surgery depended on the size of the surgical incision in the conventional operation. CONCLUSION: Laparoscopic surgery was associated with less surgical stress than open surgery and this difference was accentuated as the surgical abdominal wall trauma increased.
\end{abstract}

Key words: Adrenalectomy, Cholecystectomy, Laparoscopic surgery, Surgical stress 


\section{INTRODUCTION}

The maintenance of homeostasis is essential for life. However, this complex dynamic equilibrium is constantly threatened by endogenous or exogenous forces. Stress, defined as a state of threatened homeostasis, is limited by the adaptive response of the organism which consists of complex behavioral, neuroendocrine and cellular changes in both the central nervous system and the periphery. Surgical stress activates a composite response, which includes activation of the stress system, including its peripheral limbs, the hypothalamic-pituitary-adrenal (HPA) axis and the sympathetic nervous system as well as the renin-angiotensin axis, all changes vital for survival. ${ }^{1-5}$

The surgical wound, depending on its size, disturbs homeostasis and activates the stress system. ${ }^{1-7}$ In recent years, laparoscopic surgery has been applied both for the diagnosis and treatment of an ever expanding range of diseases, hitherto treated only by open laparotomy. The laparoscopic method has been widely accepted in the treatment of symptomatic gallstones and for removing the adrenal glands. Even though the extent of visceral surgical trauma is similar in the laparoscopic and the corresponding open surgery, the former has the advantages of a much smaller abdominal wall trauma, reduced operating time, rapid mobilization of the patient, early oral feeding, decreased hospital stay and cost as well as a decreased postoperative catabolic reaction, together with less pain and reduced need for analgesics.

The purpose of this study was to compare surgical stress in animals submitted to open and laparoscopic cholecystectomy and adrenalectomy, two operations that in their conventional forms are respectively associated with a small and large surgical incision. We studied the HPA axis by measuring plasma cortisol and the peripheral sympathetic nervous system by measuring plasma concentrations of adrenaline and noradrenaline.

\section{MATERIALS AND METHODS}

\section{Animals}

Pigs are currently the animal model of choice in many areas of biomedical research, largely because of their anatomical and physiological similarities to humans. We used large male pigs weighing about 25$30 \mathrm{Kg}$, crossbreeds of the Landrace and Large White strains. Eight of these underwent cholecystectomy, three $(n=3)$ open and the rest $(n=5)$ laparoscopic cholecystectomy. The remaining eight underwent adrenalectomy, with three $(n=3)$ of them open and the rest $(n=5)$ laparoscopic adrenalectomy.

The accommodation of the animals and their handling were in accordance with the provisions of PD 160/91 on "Protection of animals used for experimental or other scientific purposes in compliance with Council Directive 86/609/EEC.

\section{Preparation of animals}

Before the study, the pigs were acclimatized to the environment of the research centre for at least one week and before the procedures were subjected to a clinical examination by a veterinarian. Overnight fasting for 12 hours was imposed to avoid complications such as vomiting during the introduction of anesthesia and resuscitation, and gastric distension. Free intake of water was allowed up to 4-6 hours before premedication.

\section{Premedication}

The drugs given as premedication were a combination of atropine sulfate $(0.05 \mathrm{mg} / \mathrm{kg}$ i.m., Atropine, Demo), butyrophenone, azaperone (5 mg/kg i.m., Suicalm, Jansen-Cilag) and a separator anesthetic, ketamine (10 mg/kg i.m., Imalgen, Merial), which causes immobilization of the animal and heart rate reduction within 10-15 min. The administration of premedication was necessary to avoid causing extraneous stress and to manage the reluctance and potential aggression of the animals during the placing of an intravenous catheter.

\section{Intravenous access}

Through a small incision in the neck, we prepared the internal jugular vein. Under direct vision, we placed an intravenous catheter (gauge 18 or $20 \mathrm{G}$, length 2 inches), which was externalized near the right shoulder, to ensure the smooth reception of venous blood during surgery.

\section{Blood samples}

Blood samples were taken: one day before surgery, at the beginning of the operation, every 15 minutes 
during surgery and on the first postoperative morning. An aliquot of $10 \mathrm{ml}$ of blood was taken at each time point. It was divided into two (2) EDTA and two (2) heparinized tubes, placed on ice and centrifuged at $5,000 \mathrm{rpm}$ at $4^{\circ} \mathrm{C}$ for $10 \mathrm{~min}$. The plasma was stored at $-80^{\circ} \mathrm{C}$ until assayed.

\section{Anesthesia}

A vein in the dorsal surface of the earflap was catheterized with a 2 -inch 18 or 20 gauge catheter, for the administration of anesthetic. General anesthesia was preferably achieved by intravenous administration of an anesthetic in the ear vein $(2.5-3.5 \mathrm{mg} / \mathrm{kg}$ propofol - Diprivan 1\% w/v; Astra Zeneca), followed by intubation of the animal. Intubation was achieved with the animal in the supine or lateral position, using a laryngoscope with a size 1-4 Wisconsin blade, preferably in the exhalation phase; anesthesia was maintained with a volatile anesthetic (isoflurane) (Forenium, Abbott, 2-3.5\% in 2 lt Oxygen).

During the operations, vital functions were monitored by a three-lead hemodynamic monitoring device (Cardiocap CCI-104, Datex, Finland) and a pulse oximeter (OxyshuttleTM2 Criticon, Sensormedics Corp., Yorba Linda, CA, USA), attached to the ear flap.

After anesthesia induction, the left femoral artery was prepared and a catheter was placed width $20 \mathrm{G}$ (Abbott Laboratories) for connection with a pressure transducer. The latter was connected to the hemodynamic monitoring device Cardiocap CCI-104 to follow and record the blood pressure and heart rate (HR).

\section{Surgical procedures}

Surgical procedures always began between 8-9 am. They were all performed by the same surgical team at the Centre of Experimental Surgery of the Foundation for Biomedical Research of the Academy of Athens. The cholecystectomy and adrenalectomy techniques were identical between laparoscopic and open surgery.

For the cholecystectomy, the animals were placed in the supine position. Open cholecystectomy was conducted through a supra-umbilical midline incision of about $5-7 \mathrm{~cm}, 3 \mathrm{~cm}$ below the xiphoid process. Laparoscopic cholecystectomy was conducted through the creation of pneumoperitoneum (Hasson approach with a $10 \mathrm{~mm}$ subumbilical trocar and $\mathrm{CO}_{2}$ insufflation at a flow of 1lt $/ \mathrm{min}$ ). Subsequently, the following trocars were introduced under direct vision: one hypoxiphoid $(10 \mathrm{~mm})$ and one in the midclavicular line $(5 \mathrm{~mm})$. During surgery, abdominal pressure was automatically maintained at between $12-14 \mathrm{mmHg}$. Mean total surgery time was 20 minutes for open cholecystectomy and 30 minutes for laparoscopic cholecystectomy. The blood loss in both types of cholecystectomy was small, about 5-10 cc (Table 1).

Table 1. Characteristics of open and laparoscopic cholecystectomy and adrenalectomy

\begin{tabular}{lcc}
\hline & Open & Laparoscopic \\
\hline Cholecystectomy & incision of about & creation of \\
& $5-7 \mathrm{~cm}$ & pneumoperitoneum \\
& total surgery time & total surgery time \\
& $20 \mathrm{~min}$ & $30 \mathrm{~min}$ \\
Adrenalectomy & blood loss 5-10 ml & blood loss 5-10 ml \\
& incision of about & 2cm incision \\
& $20-25 \mathrm{~cm}$ & \\
& total surgery time 30 & total surgery time \\
& min & 60 min \\
& blood loss $15 \mathrm{ml}$ & blood loss $15 \mathrm{ml}$ \\
\hline
\end{tabular}

For the open adrenalectomy, the animals were placed in the supine position and a midline incision of about $20-25 \mathrm{~cm}$ was performed along the linea alba. For the laparoscopic adrenalectomy, the animals were placed in the lateral decubitus position, left side up. The table flexed to elevate the flank and the subject was secured with straps. The positioning of the animal was of utmost importance in this approach. After sterile preparation, a 2-cm incision was made $5 \mathrm{~cm}$ below the costal margin midway between the spine and xyphoid. Upon the use of a Kelly clamp and small retractors farabef, we reached the fat of the retroperitoneum. One $11 \mathrm{~mm}$ port was placed through the incision into the retroperitoneum and a $10 \mathrm{~mm}$ camera was placed through the port to ensure correct positioning, carbon dioxide was initiated with a maximum pressure of $15 \mathrm{mmHg}$. Another $10 \mathrm{~mm}$ port was placed under direct vision along the costal margin anterior to the initial trocar; a laparoscopic grasper was placed through the port and used to ensure adequate working space. Two additional 5 $\mathrm{mm}$ ports were placed under direct vision along the 
costal margin with orientation towards the location of the adrenal gland, one anterior to the initial trocar and one interior to these. All ports were located in the retroperitoneal space. The average operating time of open and laparoscopic adrenalectomy was 30 and $60 \mathrm{~min}$, respectively. Minimal access operations like laparoscopic adrenalectomy require significantly more time to complete than their conventional counterparts (Table 1$)$.

\section{Recovery}

During recovery, the pigs were placed in a warm environment $\left(30^{\circ} \mathrm{C}\right)$, with appropriate wall facing to ensure their safety. Their vital functions were monitored continuously to prevent complications. We inspected the surgical site and all catheters placed. Upon receipt of all samples, the animals were sacrificed by intravenous administration of pentobarbital.

\section{Methods of measuring hormones in blood plasma}

The measurement of cortisol in plasma was based on the chemiluminescent enzyme immunoassay method and performed using a kit by Roche Diagnostics LTD at the Elecsys 2010 analyser, according to the manufacturer's instructions.

The measurement of plasma catecholamines was performed using High Pressure Liquid Chromatography (HPLC) followed by electrochemical detection. The separation was the result of the combined action of a stationary and a mobile phase. The sample was introduced at the top of the column and, with the help of the mobile phase, catecholamine fractions moved in the form of bands, eventually released sequentially for electrochemical measurement.

\section{Statistical analysis}

All laboratory measurements of hormones were conducted in duplicate. Results are expressed as mean \pm SEM. Both parametric and non-parametric tests were performed.

\section{RESULTS}

\section{Survival, morbidity, complications}

After taking all blood samples, the animals were euthanized by administration of a large dose of pentobarbital 24 hours after surgery. None of the animal models undergoing cholecystectomy and adrenalec- tomy, either laparoscopically or open, showed any complications (fever, bleeding, respiratory failure) during the postoperative period or died until the time of euthanasia.

\section{Cholecystectomy}

Small but nonsignificant differences were observed in plasma cortisol values between open and laparoscopic cholecystectomy (Figure 1). The values of cortisol in laparoscopic surgery were lower than the values in the open operations at the same operative times of 0,15 and $30 \mathrm{~min}$ and similar in the first postoperative day (d1). During laparoscopic surgery, we noted a gradual increase of plasma cortisol, which, however, was clearly lower than the increase in cortisol observed in the open cholecystectomy.

Plasma catecholamine concentrations were significantly different between open and laparoscopic cholecystectomy. The levels of plasma adrenaline in the laparoscopic group were significantly lower than those obtained in open surgery at operative times $0,15,30 \mathrm{~min}$, and on d1. Statistical differences between the open and laparoscopic surgery groups were observed at operative times 0 and $15 \mathrm{~min}$, with plasma adrenaline being lower in the laparoscopic than the open cholecystectomy (Figure 2, $\mathrm{P}<0.05$ ). During open surgery, we noted a gradual decrease of plasma adrenaline, in contrast to laparoscopic surgery, where the value of adrenaline remained approximately stable. On the first postoperative

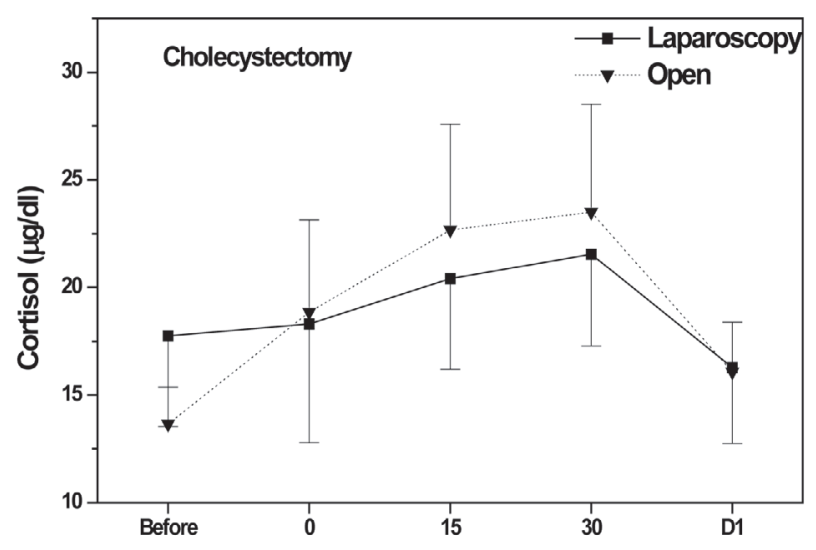

Figure 1. Plasma cortisol during open and laparoscopic cholecystectomy. Values are expressed as Mean \pm SEM. Before: one day before surgery, 0: incision, 15, 30: minutes during surgery, D1: first postoperative morning. 


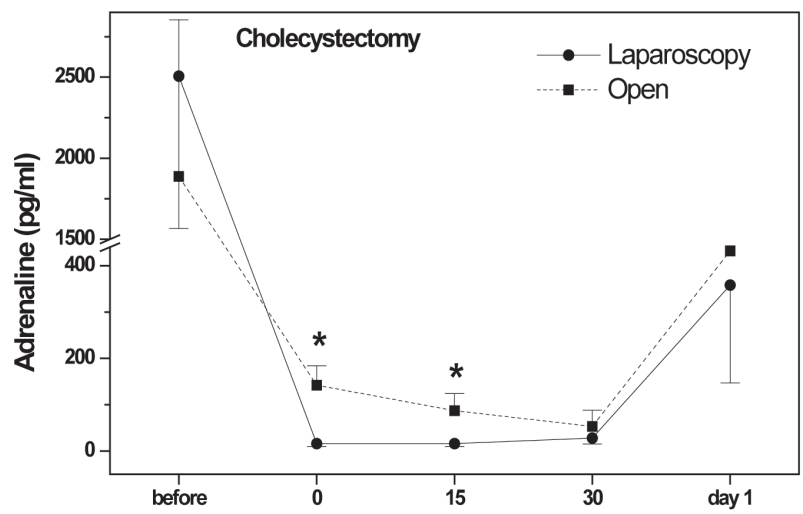

Figure 2. Plasma adrenaline during open and laparoscopic cholecystectomy. Values are expressed as Mean \pm SEM. *depicts statistical significance $(\mathrm{P}<0.05)$. Before: one day before surgery, 0: incision, 15, 30: minutes during surgery, D1: first postoperative morning.

day, plasma adrenaline values in both the open and laparoscopic cholecystectomy groups were lower than the corresponding preoperative values.

Noradrenaline values in laparoscopic surgery were lower than with the open procedure at operative times 0 and $15(\mathrm{P}<0.05)$, while they were similar at 30 $\min$. On the first postoperative day, the value of noradrenaline in the laparoscopic group was higher than the corresponding value in the open group (Figure 3 ). A statistically significant difference was observed only at time 0 , where the value of noradrenaline in the laparoscopic group was lower than in the open group (Figure 3, $(\mathrm{P}<0.05)$ ).

\section{Adrenalectomy}

We observed significant differences in plasma cortisol values between open and laparoscopic unilateral adrenalectomy (Figure 4). Plasma cortisol in laparoscopic surgery was significantly lower compared to open operations both at operative times 0,15 and $30 \mathrm{~min}$ and on the first postoperative day $(\mathrm{P}<0.05)$. The highest difference, observed at time 0 , gradually decreased at times 15 and $30 \mathrm{~min}$.

As with cortisol, circulating adrenaline and noradrenaline concentrations were significantly lower during laparoscopic than during open adrenalectomy. The values of plasma adrenaline in laparoscopic surgery were significantly lower than those in the open operations at operative times of 0,15 and 30

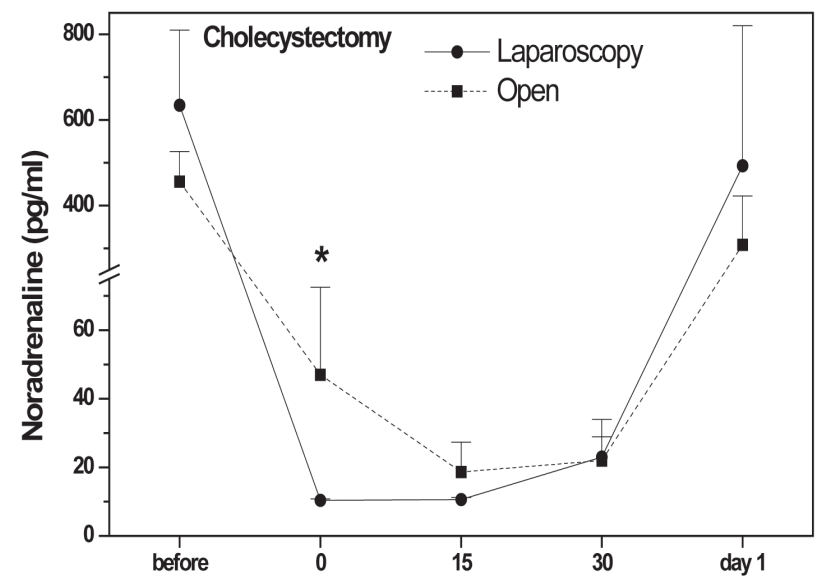

Figure 3. Plasma noradrenaline during open and laparoscopic cholecystectomy. Values are expressed as Mean \pm SEM. *depicts statistical significance $(\mathrm{P}<0.05)$. Before: one day before surgery, 0: incision, 15, 30: minutes during surgery, day 1: first postoperative morning.

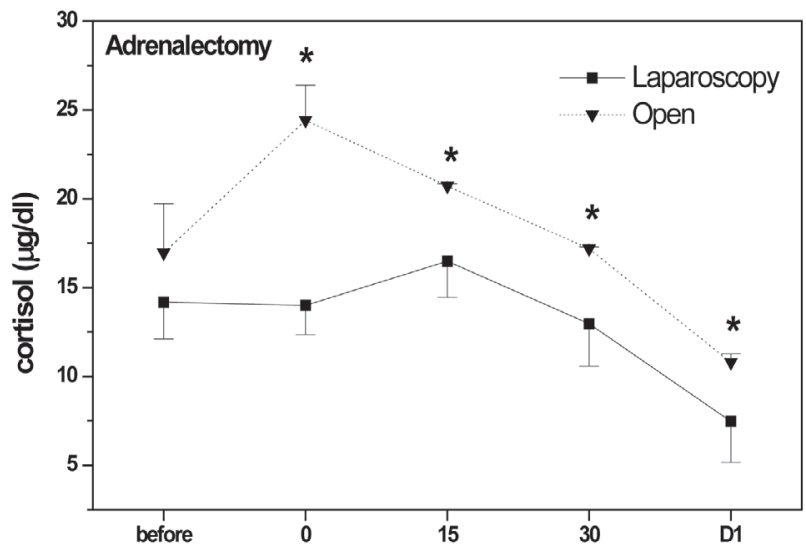

Figure 4. Plasma cortisol during open and laparoscopic adrenalectomy. Values are expressed as Mean \pm SEM. *depicts statistical significance $(\mathrm{P}<0.05)$. Before: one day before surgery, 0: incision, 15, 30: minutes during surgery, D1: first postoperative morning.

$\min (\mathrm{P}<0.01)$, while, in contrast, they were lower in open adrenalectomy than in laparoscopic surgery on the first postoperative day (d1) (Figure 5). During open surgery we noted a gradual increase of plasma adrenaline (which peaked at $30 \mathrm{~min}$ ), in contrast to laparoscopic surgery, where it remained relatively stable, with a slight increase at $30 \mathrm{~min}$. However, while in laparoscopic adrenalectomy adrenaline values remained approximately stable on day 1 , in open adrenalectomy they decreased dramatically postoperatively. 


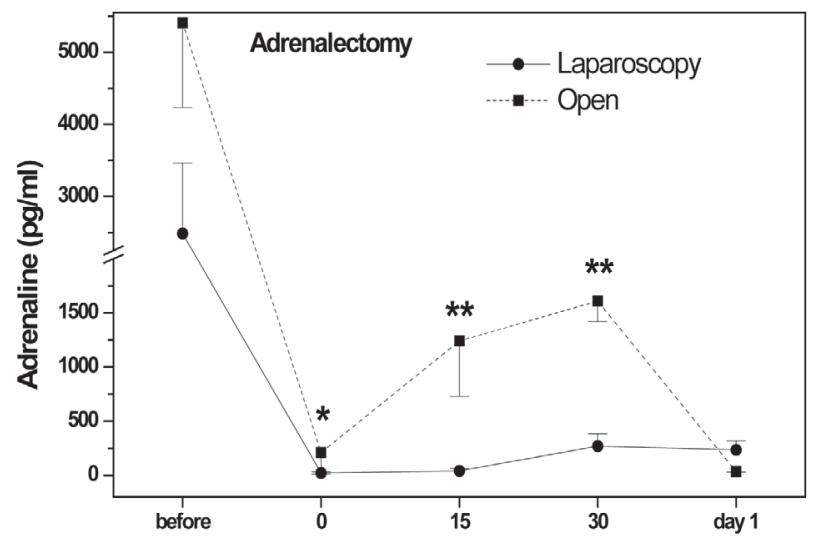

Figure 5. Plasma adrenaline during open and laparoscopic adrenalectomy. Values are expressed as Mean \pm SEM. *, ** depict statistical significance $(\mathrm{P}<0.05, \mathrm{P}<0.01$ respectively $)$. Before: one day before surgery, 0: incision, 15, 30: minutes during surgery, day 1: first postoperative morning.

The variation of plasma noradrenaline values between the two types of surgery was generally similar to that observed for adrenaline values (Figure 6, $\mathrm{P}<0.01$ ).

\section{Hemodynamic parameters}

An analysis of the measurements of hemodynamic parameters (heart rate, systolic and diastolic blood pressure, mean arterial pressure, body temperature and hemoglobin saturation in $\mathrm{O}_{2}$ ) revealed small but significant differences between open and laparoscopic cholecystectomy (Table 2). During laparoscopic surgery, mean arterial pressure was significantly lower than in the open procedure. In laparoscopic surgery, we also noted a more significant drop in the heart rate than in the open procedure (Table 1). In addition, the acute distension of the peritoneal cavity, induced by the injection of $\mathrm{CO}_{2}$, resulted in elevation of the diaphragm and most likely an increase of heart pressure and, thus, a greater increase in heart rate and

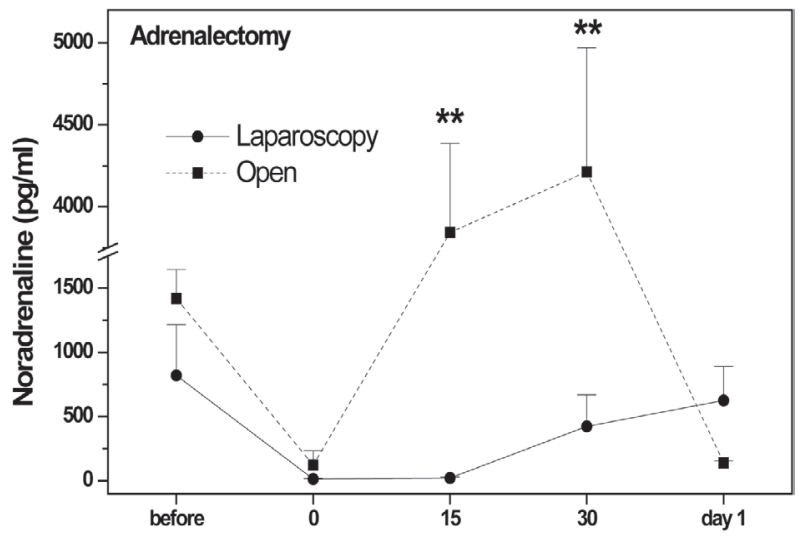

Figure 6. Plasma noradrenaline during open and laparoscopic adrenalectomy. Values are expressed as Mean \pm SEM. ** depicts statistical significance $(\mathrm{P}<0.01)$. Before: one day before surgery, 0: incision, 15, 30: minutes during surgery, day 1: first postoperative morning.

mean blood pressure than in the open procedure, where the peritoneal cavity was not dilated at all at 0 minutes.

We observed an increased heart rate and blood pressure during the preparation and removal of the adrenal gland in both open surgery (approximately 15 minutes, Table 2) and endoscopic adrenalectomy (30 and 45 minutes). The mean blood pressure (MP) in endoscopic adrenalectomy was maintained at elevated levels compared to the open surgery method.

From the analysis of the hemodynamic parameters - heart rate, systolic and diastolic blood pressure, mean arterial pressure, body temperature - a slight but significant difference between the open and endoscopic adrenalectomy was observed. Acute dilatation of the retroperitoneal space that was created with administering $\mathrm{CO}_{2}$, resulted in the elevation of the diaphragm and a consequent increase in heart

Table 2. Mean hemodynamic parameters during surgery

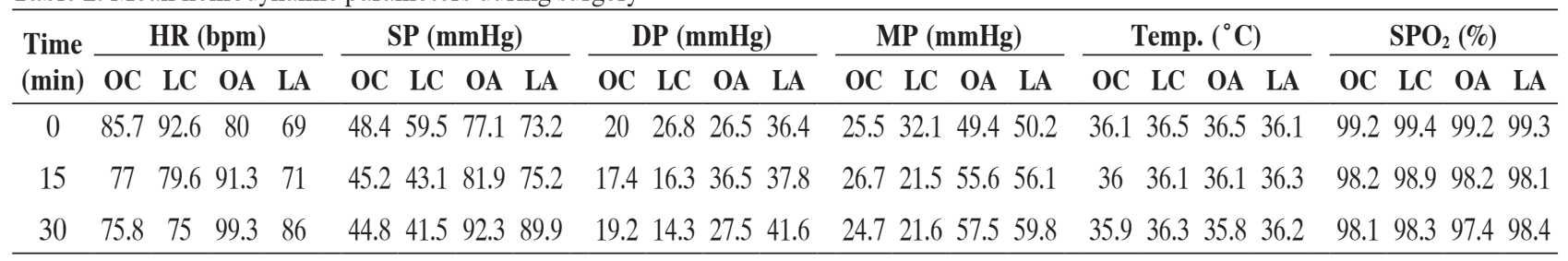

HR: heart rate, SP: systolic blood pressure, DP: diastolic blood pressure, MP: mean arterial pressure, Temp.: body temperature, $\mathrm{SPO}_{2}$ : hemoglobin saturation in $\mathrm{O}_{2}$. OC: open cholecystectomy, LC: laparoscopic cholecystectomy, OA: open adrenalectomy, LA: laparoscopic adrenalectomy. 
pressure. This resulted in a significant increase in heart rate and mean blood pressure in the laparoscopic surgery (operative time 0 minutes) compared to the open surgery, where the peritoneal cavity was not dilated at all.

\section{DISCUSSION}

Early studies of hormonal responses to surgical stress focused on the study of the HPA axis by measuring circulating $\mathrm{CRH}$, AVP, corticotropin (ACTH) and corticosterone or cortisol, the renin-angiotensinaldosterone system by measuring renin (PRA) and aldosterone (Aldo), as well as the sympathetic nervous system by measuring the catecholamines adrenaline and noradrenaline. ${ }^{1-5}$ Several additional hormones were proposed for measuring the body's immune and metabolic response to surgery, such as IL-1beta, IL-6, IL-8 and others. ${ }^{6-9}$ The main and most reliable peripheral hormones that correlated well with the extent of the surgical trauma were cortisol and the catecholamines. In this light, this study investigated the suitability of these hormones for reflecting the body's response to the wounds of open and laparoscopic cholecystectomy and adrenalectomy in an animal model.

Morbidity, mortality and the time of recovery and full return of patients undergoing surgical procedures to their usual activities are due to and depend not only on the presence or absence of intraoperative complications, but also on the severity of the surgical wound which also determines the body's stress response. ${ }^{10,11}$ The severity of an abdominal surgical wound is determined by the length of the incision in the skin and the other layers of the abdominal wall, ${ }^{12,13}$ the size of the parietal peritoneal trauma ${ }^{14,15}$ and the manipulations within the peritoneal cavity, mainly in the intestine..$^{12,16} \mathrm{An}$ important role is also played by dehydration and heat loss due to the exposure of the intra-abdominal viscera to the environment during laparotomy. Opinions on the degree and importance of each of the above factors are divided..$^{13,16}$

The trauma of open cholecystectomy is generally small. The operative time is short, the incision limited and there are few manipulations in the peritoneal cavity. Thus, the related stimuli for the body's response are small, with no excessive dehydration, heat loss and/or blood loss.
Laparoscopic cholecystectomy, on the other hand, further reduces the surgical trauma, replacing the surgical incision with four small incisions, while it decreases intraperitoneal manipulations. ${ }^{15}$ Therefore, the stress response of the body should be more limited. The above view is reinforced by the spectacular results of faster mobilization, nutrition, recovery and the patient's return to his or her normal daily activities.

Other authors have argued that the trauma of laparoscopic cholecystectomy, although lacking the long incision in the abdominal wall, is ultimately no smaller than that of open surgery and thus the body's response is practically the same. They have suggested that: a) the tissue trauma in the form of intraperitoneal manipulations - pulling of the gallbladder and mesentery - are similar in both methods ${ }^{17}$ and b) the pneumoperitoneum is an aggravating factor on the surgical wound, because: 1) it causes acute dilatation of the peritoneum and irritation of the peritoneal nerve endings that activates receptors, permanent pressure on the diaphragm during surgery, $\mathrm{CO}_{2}$ absorption by the peritoneum, increased partial pressure in the blood and respiratory acidosis ${ }^{17,18}$ and 2) the commercial $\mathrm{CO}_{2}$ used for creating the pneumoperitoneum is fluid and changes into its gaseous form at its boiling point of $21.1^{\circ} \mathrm{C} .{ }^{19}$ Thus, its prolonged use might cause a drop in body temperature, even though this is at least partially avoided by heating it at 30 to $30.5^{\circ} \mathrm{C} .{ }^{20}$

Other studies have suggested the effect of the pneumoperitoneum is not a major wound-aggravating factor because: a) a significant increase in $\mathrm{CO}_{2}$ and respiratory acidosis in laparoscopic cholecystectomy is observed only in patients with chronic cardiopulmonary failure and preoperative restrictive lung function, ${ }^{21-24} \mathrm{~b}$ ) with increasing surgical experience, the pneumoperitoneum is sufficient even at smaller pressures, ${ }^{1,4,6}$ and c) the duration of surgery is now considerably briefer than that described in the earlier series. $^{6}$

Based on the data of this study, we propose the hypothesis that the creation and maintenance of a pneumoperitoneum may be partially responsible for the small differences in cortisol and catecholamine values observed between laparoscopic and open cholecystectomy. Abdominal distension, diaphragmatic pressure on the heart, the absorption of $\mathrm{CO}_{2}$ and the partial increase of $\mathrm{PCO}_{2}$ in the blood may play roles 
as stressors that equal the stress of opening of the abdominal wall. In open cholecystectomy, the incision was just $5-7 \mathrm{~cm}$ in length and went only through the skin, the subcutaneous tissue and the peritoneum, as it was performed in the hypoxyphoid region, along the linea alba. It was not an oblique subpleural incision, which involves larger and stronger muscle groups, thus potentially creating more stress.

The systemic response to surgical injury is complex and involves psychological and physical stress and neuroendocrine and inflammatory mediators. In humans, there is anticipatory stress that is associated with elevations of cortisol and catecholamines on the day of surgery. The anesthetic management has also been related to the level of surgical stress of the patient. Similarly, the type of ventilation strategy, administration of sufficient pain relief, adequate resuscitation and the utilization of rapid-onset and short-acting volatile anesthetic agents are all an integral part of the surgical stress response. ${ }^{25}$

The similarity of catecholamine and cortisol responses between laparoscopic and open cholecystectomy during the first postoperative day was unexpected because in humans laparoscopic cholecystectomy requires less analgesics, produces less ileus and is characterized by faster recovery, suggesting decreased surgical stress than in the open procedure.

In unilateral adrenalectomy, cortisol, adrenaline and noradrenaline were significantly higher in the group of animals that underwent open rather than laparoscopic surgery during the operative period. Our results here clearly show that open is more stressful than laparoscopic adrenalectomy, but less so in the immediate postoperative period. To avoid influencing the study of surgical stress by different surgical skills, the entire experimental study was done by two and the same surgeons.

The body's stress response in the two different types of surgery, open vs. laparoscopic, has been studied to some extent; however, there is considerable disagreement in the existing literature. Reduced adrenaline and noradrenaline values after laparoscopic cholecystectomy were reported in earlier studies and this is consistent with our controlled study. ${ }^{6,15,23,26,29} \mathrm{On}$ the other hand, when examining the HPA axis, the majority of earlier studies reported great differences in cortisol levels between laparoscopic and open cho- lecystectomies, ${ }^{8,9,15,26,29,30}$ while a few showed no such differences. ${ }^{7,17}$ Our well controlled study confirms the results of the latter and our explanation for this is that the stress of the small surgical incision is not greater that the stress of the laparoscopy incisions, the pneumoperitoneum, etc. of the laparoscopic surgery.

Endoscopic adrenalectomy is one of the most "minimally invasive" techniques of modern surgery. It has been distinguished according to the access as retroperitoneal endoscopic adrenalectomy or as laparoscopic adrenalectomy when using the transabdominal approach by the creation of pneumoperitoneum. ${ }^{31,32}$

From 1992, when the first patient with an adrenocortical adenoma causing Cushing syndrome had laparoscopic adrenalectomy, it has emerged as the surgery of choice for the treatment of benign, functional and non-adrenal tumors. ${ }^{33}$ It has spread widely, unlike retroperitoneal endoscopic adrenalectomy. This is partly due to the improvement of laparoscopic tools, but mainly to the fact that this type of laparoscopic surgery is accompanied by very little blood loss, low morbidity, short hospital stay, rapid return to normal physical activity and work in combination with the excellent esthetic results and much fewer postoperative hernias in comparison with open adrenalectomy. ${ }^{34,35}$

Aldosteromas are the most frequent indication for laparoscopic adrenalectomy and account for one third of such procedures performed today. Cortisolsecreting adrenocortical adenomas causing Cushing syndrome represent a smaller percentage. Laparoscopic adrenalectomy is applied in most but not all cases of pheochromocytomas, mainly for technical reasons. ${ }^{36}$

Additional studies with a larger number of experimental models, which will confirm that the body's metabolic and neuroendocrine response is more limited in laparoscopic than in open, cholecystectomy and adrenalectomy, are awaited. We conclude that the systemic stress response, as assessed by determining the levels of serum catecholamines and cortisol in animal models undergoing cholecystectomy and adrenalectomy, is significantly reduced in the laparoscopic compared to the open procedure. ${ }^{35}$ During laparoscopic surgery, the pneumoperitoneum appears to be a major stressor comparable to that of surgical incisions and the manipulations made by the surgeon during open surgery. 


\section{REFERENCES}

1. Udelsman R, Chrousos GP, 1988 Hormonal responses to surgical stress. Adv Exp Med Biol 245: 265-272.

2. Giuffre KA, Udelsman R, Listwak S, Chrousos GP, 1988 Effects of immune neutralization of corticotropin-releasing hormone, adrenocorticotropin, and beta-endorphin in the surgically stressed rat. Endocrinology 122: 306-310.

3. Udelsman R, Goldstein DS, Loriaux DL, Chrousos GP, 1987 Catecholamine-glucocorticoid interactions during surgical stress. J Surg Res 43: 539-545.

4. Udelsman R, Norton JA, Jelenich SE, et al, 1987 Responses of the hypothalamic-pituitary-adrenal and renin-angiotensin axes and the sympathetic system during controlled surgical and anesthetic stress. J Clin Endocrinol Metab 64: 986-994.

5. Chrousos GP, 2009 Stress and Disorders of the Stress System. Nat Rev Endocrinol 5: 374-381.

6. Glaser F, Sannwald G, Buhr H, et al, 1995 General stress response to conventional and laparoscopic cholecystectomy. Ann Surg 221: 372-380.

7. Jakeways MS, Mitchell V, Hashim IA, et al, 1994 Metabolic and inflammatory responses after open or laparoscopic cholecystectomy. Br J Surg 81: 127-131.

8. Mansour A, Stiegman GV, Yamamoto M, Berguer R, 1992 Neuroendocrine stress response after minimally inavasive surgery in pigs. Surg Endosc 6: 294-297.

9. Marcovich R, Antoinette L, Williams BS, Seifman BD, Wolf SJ Jr, 2001 A Canine Model to Assess the Biochemical Stress Response to Laparoscopic and Open Surgery. J Endour 15: 1005-1008.

10. Douglas RG, Shaw JHF, 1989 Metabolic response to sepsis and trauma. Br J Surg 76: 115-122.

11. Gann DS, Ameral JF 1989 Endocrine and metabolic responses to injury. In Schwarz S, Shires G, Spencer F (eds) Principles of Surgery, Fifth Ed, Mc Graw-Hill Book Company, Vol.1, pp, 1-69.

12. Bailey RW, Zucker KA, Flowers JL, Scovill WA, Graham SM, Imbembo AL, 1991 Laparoscopic cholecystectomy. Experience with 375 consecutive patients. Ann Surg 214: 531-541

13. Schauer PR, Luna J, Ghiatas AA, Glen ME, Warren JM, Sirinek KR, 1993 Pulmonary function after laparoscopic cholecystectomy. Surgery 114: 389-399.

14. Deuss U, Dietrich J, Kaulen D, et al, 1994 The stress response to laparoscopic cholecystectomy: investigation of endocrine parameters. Endoscopy 26: 235-238.

15. Donald RA, Perry EG, Wittert GA, et al, 1993 The plasma ACTH, AVP, CRH and catecholamine responses to conventional and laparoscopic cholecystectomy. Clin Endocrinol 38: 609-615.

16. Schippers E, Ottinger AP, Anurov M, Polivoda M, Schumpelick V, 1993 Laparoscopic cholecystectomy: A minor abdominal trauma? World J Surg 17: 539-542.

17. Milheiro A, Sousa FC, Manso EC, Leitao F, 1994 Metabolic responses to cholecystectomy: open vs laparoscopic approach. J Laparoendosc Surg 4: 311-317.

18. Cooper GM, Scoggins AM, Ward ID, Murphy D, 1982 Laparoscopy: a stressful prosedure. Anesthesia 37: 266-269.
19. Ott DE, 1991 Laparoscopic hypothermia. J Laparoendosc Surg 1: 127-131.

20. Ott DE, 1991 Correction of laparoscopic insufflation on hypothermia. J Laparoendosc Surg 1: 183-186.

21. Frazee RC, Roberts JW, Okeson GC, et al, 1991 Open Versus Laparoscopic Cholecystectomy. A comparison of postoperative pulmonary function. Ann Surg 213: 651-653.

22. Johnston D, Litwin D, Osachoff J, et al, 1992 Postoperative respiratory function after laparoscopic cholecystectomy. Surg Laparosc Endosc 2: 221-226.

23. Schauer PR, Sirenek KR, 1995 The laparoscopic approach reduces the endocrine response to elective cholecystectomy. Am Surg 61: 106-111.

24. Wittgen C, Naunheim KS, Andrus CH, Kaminski DL, 1993 Preoperative Pulmonary Function Evaluation for laparoscopic cholecystectomy. Arch Surg 128: 880-886.

25. Wilmore DW, 2002 From Cuthbertson to fast-track surgery: 70 years of progress in reducing stress in surgical patients. Ann Surg 236: 643-649.

26. Le Blanc-Louvry I, Coquerel A, Koning E, Maillot C, Ducrotte P, 2000 Operative stress response is reduced after laparoscopic compared to open cholecystectomy: the relationship with postoperative pain and ileus. Dig Dis Sci 45: 1703-1713.

27. Crema E, Ribeiro EN, Hial AM, Alves JT, Jr, Pastore R, Silva AA, 2005 Evaluation of the response of cortisol corticotropin and blood platelets kinetics after laparoscopic and open cholecystectomy. Acta Cir Bras 20: 364-370.

28. Ortega AE, Peters JH, Incarbore R, et al, 1996 A prospective randomized comparison of the metabolic and stress hormonal responses of laparoscopic and open cholecystectomy. J Am Coll Surg 183: 249-256.

29. Karayiannakis AJ, Makri GG, Mantzioka A, Karousos D, Karatzas G, 1997 Systemic stress response after laparoscopic or open cholecystectomy: a randomized trial. Br J Surg 84: 467-471.

30. Luo K, Li JS, Li LT, Wang KH, Shun JM, 2003 Operative stress response and energy metabolism after laparoscopic cholecystectomy compared to open surgery. World J Gastroenterol 9: 847-850.

31. Lacroix A, Tremblay J, Rousseau G, Bouvier M, Hamet P, 1997 Propranolol therapy for ectopic beta-adrenergic receptors in adrenal Cushing's syndrome. New Engl J Med 337: 1429-1434.

32. Russell CF, Hamberger B, van Heerden JA, Ilstrup DM, 1982 Adrenalectomy: anterior or posterior approach? Am J Surg 144: 322-324.

33. Gagner M, Lacroix A, Bolte E, 1992 Laparoscopic adrenalectomy in Cushing's syndrome and pheochromocytoma. N Engl J Med 327: 1033.

34. Higashihara E, Tanaka Y, Horie S, et al, 1992 A case report of laparoscopic adrenalectomy. Nippon Hinyokika Gakkai Zasshi 83: 1130.

35. Mercan S, Seven R, Ozarmagan S, Tezelaman S, 1995 Endoscopic retroperitoneal adrenalectomy. Surgery (St. Louis) 118: 1071-1076.

36. Prinz R, 1995 A comparison of laparoscopic and open adrenalectomies. Arch Surg 130: 489-494. 